
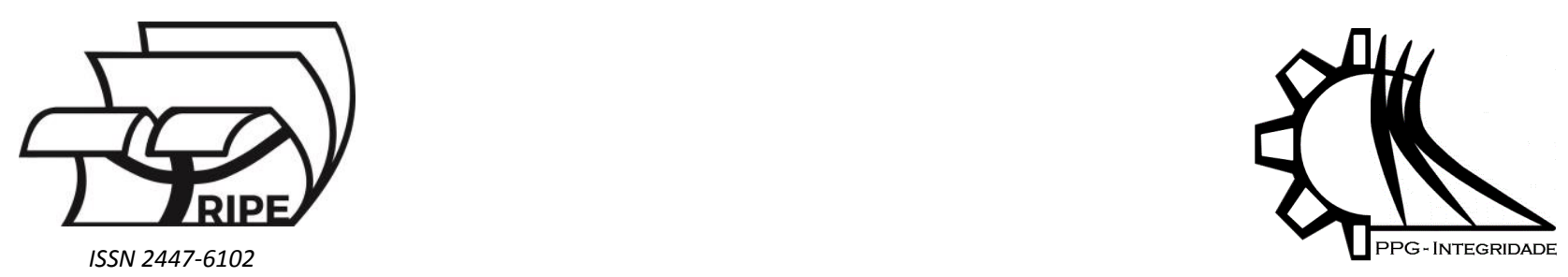

Article

\title{
Desenvolvimento de um modelo numérico para a predição de um escoamento em bocal do tipo H.O.M.E.R
}

\author{
El Halal, Y.B. ${ }^{1}$, Marques, C.H . ${ }^{2}$, Rocha, L.A.O ${ }^{3}$, Isoldi, L.A. ${ }^{4}$, Lemos, R.L. ${ }^{5}$ and Dos Santos, E.D. ${ }^{6, *}$ \\ 1 Escola de Engenharia, Universidade Federal do Rio Grande - FURG, Av. Itália, km 8, Rio Grande - RS, 96201-900, Brazil; \\ youssefhalal20@gmail.com \\ 2 Escola de Engenharia, Universidade Federal do Rio Grande - FURG, Av. Itália, km 8, Rio Grande - RS, 96201-900, Brazil; \\ cristoferhood@furg.br \\ 3 Programa de Pós-Graduação em Engenharia Mecânica, Universidade do Vale do Rio dos Sinos - UNISINOS, São Leopoldo, RS, \\ Brazil, luizor@unisinos \\ 4 Programa de Pós-Graduação em Engenharia Oceânica, Universidade Federal do Rio Grande - FURG, Av. Italia, km 8, Rio Grande \\ - RS, 96201-900, liercioisoldi@furg.br \\ 5 Programa de Pós-Graduação em Engenharia Oceânica, Universidade Federal do Rio Grande - FURG, Av. Italia, km 8, Rio Grande \\ - RS, 96201-900, er.lemos@outlook.com \\ 6 Programa de Pós-Graduação em Engenharia Oceânica, Universidade Federal do Rio Grande - FURG, Av. Italia, km 8, Rio Grande \\ -RS, 96201-900, elizaldosantos@furg.br \\ * Correspondence: elizaldosantos@furg.br; Tel.: +55-53-3233-6916 (E.D.S.)
}

Received: 14/12/2018; Accepted: 05/01/2019; Published: 06/02/2019

Resumo: No presente estudo é desenvolvido um modelo numérico para a abordagem de escoamentos turbulentos no regime permanente em bocais do tipo H.O.M.E.R (do inglês: High-speed Orienting Momentum with Enhanced Reversibility) que consiste na mistura de dois jatos incidentes sobre superfícies de Coanda. Essa superfície causa uma deflexão no escoamento permitindo que o bocal atue como um dispositivo de manobra em aplicações aeronáuticas. O principal objetivo é avaliar o modelo numérico desenvolvido comparando com resultados numéricos da literatura. As equações de conservação de massa e quantidade de movimento médias no tempo são resolvidas numericamente através do método de volumes finitos. Para resolver o problema do fechamento da turbulência foi empregada modelagem clássica (RANS - do inglês: Reynolds Averaged Navier Stokes) com modelo $k-\varepsilon$. Primeiramente, um teste de independência de malha será realizado, com o intuito de dispender menos recursos computacionais e obter resultados precisos. Em seguida, serão feitas as simulações em regime permanente, com o objetivo de obter os ângulos de deflexão $(\alpha)$ gerados com a diferença das vazões mássicas injetadas em cada um dos canais do bocal H.O.M.E.R $\left(m^{*}\right)$. Posteriormente, esses resultados são comparados com os obtidos numericamente na literatura. Os resultados obtidos tiveram o mesmo comportamento obtido na literatura, onde o aumento da diferença entre os jatos de entrada conduziu a um aumento no ângulo de deflexão do jato no bocal. Com exceção de um valor específico $\left(m^{*}=0.2\right)$ os resultados obtidos no presente trabalho apresentaram uma boa concordância com os preditos numericamente na literatura.

Palavras-chave: Estudo Numérico, Escoamento Turbulento, Efeito Coanda, Bocal H.O.M.E.R.

\section{Development of a numerical model for prediction of a flow in a H.O.M.E.R nozzle}

Abstract: In the present study a numerical model is developed for the approach of turbulent flows in the permanent regime in HOMER (High-speed Orienting Momentum with Enhanced Reversibility) nozzles consisting of the mixing of two jets and incidence on a surface of Coanda. This surface causes a deflection in the flow allowing the nozzle to act as a maneuvering device in aeronautical applications. The main objective is to evaluate the numerical model 
developed comparing with numerical results of the literature. The time-averaged mass and momentum conservation equations are solved numerically by the finite-volume method. To solve the problem of turbulence closure, classical model (RANS) was used with model $k-\varepsilon$. Firstly, a mesh independence test will be performed with the aim of expending less computational resources, while achieving accurate results. Then, the simulations will be done in a steady state, with the objective of obtaining the deflection angles $(\alpha)$ generated with the difference of the injected mass flow in each of the channels of the H.O.M.E.R $\left(\mathrm{m}^{*}\right)$ nozzle. Subsequently, these results are compared with those obtained numerically in the literature. The results obtained had the same behavior as those obtained in the literature, in which the increase in the difference between the incoming jets led to an increase in the angle of jet deflection in the nozzle. With the exception of a specific value $\left(m^{*}=0.2\right)$ the results obtained in the present work presented a good agreement with those predicted numerically in the literature.

Keywords: Numerical Study, Turbulent Flow, Coanda Effect, Nozzle H.O.M.E.R.

\section{Introdução}

Nos últimos anos, a busca por máquinas mais eficientes vem aumentando (Rose et al., 2001; Johnson, 2006), pois, aumentar a eficiência significa reduzir o consumo de combustíveis fósseis, onde, por sua vez, acarreta em menores custos e menores emissões de gases de efeito estufa. Estudos afirmam que no ano de 2000, 28\% dos gases estufa eram emitidos por meios de transporte e, dessa porcentagem, $5 \%$ são emitidos por transporte aéreo (Chapman, 2007).

A indústria aeroespacial é de suma importância no cenário econômico e social pelo transporte de mercadorias e pessoas em tempos reduzidos quando comparados a outros meios de transportes. Nesse contexto, vários estudos têm sido desenvolvidos buscando melhorar a eficiência dos sistemas de propulsão. Entre eles, o projeto ACHEON (Aerial Coanda High Efficiency Orienting-jet Nozzle) vem desenvolvendo um novo sistema de propulsão baseado no efeito de Coanda para amplificação do escoamento. O projeto possui duas tecnologias, a primeira é o bocal do tipo H.O.M.E.R. (do inglês: High-speed Orienting Momentum with Enhanced Reversibility), a segunda é o PEACE (do inglês: Plasma Enhanced Actuator for Coanda Effect) (Dumas et al., 2012). O presente trabalho irá se concentrar apenas no estudo do bocal do tipo H.O.M.E.R., maiores informações sobre o PEACE podem ser encontradas na literatura (Rodrigues et al., 2013).

O bocal H.O.M.E.R. possui dois canais, um superior e um inferior, separados por um septo central. Dois jatos com uma velocidade pré-determinada são então forçados nesses canais. Esses jatos passam através de superfícies curvas, sendo então acelerados. O jato com maior velocidade, tende a arrastar o outro consigo, gerando uma deflexão do escoamento (Dumas et al., 2012). No caso de velocidades iguais, não haverá deflexão. O fenômeno descrito é conhecido como Efeito Coanda e tem esse nome devido a Henry Coanda, que em 1936, patenteou o primeiro dispositivo que utiliza desse fenômeno para funcionar (Coanda,1936). O sistema em questão, promete entregar às aeronaves maior manobrabilidade e reduzir as distâncias de pouso e decolagem através de propulsão vetorial (Pascoal et al, 2013).

Por ser um sistema ainda em desenvolvimento, apenas poucas análises do mesmo são feitas numericamente em pacotes de CFD (do inglês: Computational Fluid Dynamics), de modo que estudos de verificação desses modelos são escassos. O objetivo do presente trabalho é desenvolver um modelo numérico para estimar o escoamento em bocais do tipo H.O.M.E.R. Os resultados obtidos para o ângulo de deflexão em função da diferença de vazão mássica entre os bocais foram comparados com os preditos em Trancossi e Dumas (2011). Em todos os casos foram considerados escoamentos turbulentos, incompressíveis, bidimensionais e no regime permanente. Para a abordagem do escoamento turbulento foi considerada a modelagem clássica da turbulência (RANS - do inglês: Reynolds Averaged Navier Stokes) com modelo $k-\varepsilon$ para estimativa da viscosidade turbulenta usada no fechamento das equações pelo processo de média temporal (Launder e Spalding, 1972). As equações de conservação de massa e quantidade de movimento médias no tempo foram resolvidas numericamente através do método de volumes finitos (Versteeg e Malalasekera, 2007).

\section{Modelagem matemática e numérica}

Como se trata da verificação de um modelo numérico, serão realizadas várias simulações numéricas com um domínio computacional que simula o bocal de Coanda do tipo H.O.M.E.R. O domínio em questão é o mesmo empregado no trabalho de Trancossi e Dumas (2011). 
A Figura 1a mostra a geometria que será utilizada no problema. O escoamento é decorrente da imposição de duas vazões mássicas nas duas entradas do dispositivo, como mostra a Fig. 1b. No canal superior do dispositivo, uma vazão mássica $\dot{m}_{1}$ é forçada ao longo do canal, ao mesmo tempo que uma vazão mássica $\dot{m}_{2}$ é forçada no canal inferior. A soma dessas vazões mássicas será sempre igual a $8 \mathrm{~kg} / \mathrm{s}$, porém, com o objetivo de verificar as deflexões causadas pela diferença de velocidade do fluido, essas vazões serão variadas, como mostra a Tab. 1. Tanto no canal superior quanto no canal inferior, as vazões mássicas serão injetadas com uma sobrepressão de 4000 Pa. As entradas são separadas por uma região hachurada denominada de septo. Na junção entre os dois jatos de entrada é inserido um bocal com superfícies de Coanda que causam uma aceleração do escoamento e deflexão no jato misturado. Essa deflexão é dependente da diferença de magnitude imposta nas duas entradas e é representada por $\alpha$ na Fig. $1 \mathrm{~b}$. Também, na Fig. 1, as paredes onde são impostas a condição de não-deslizamento e impermeabilidade são hachuradas.

Nesse estudo foi utilizado ar como fluido de trabalho com massa específica ( $\rho$ ) igual a $1,225 \mathrm{~kg} / \mathrm{m}^{3}$ e viscosidade dinâmica $(\mu)$ igual a $1,7894 \times 10^{-5} \mathrm{~kg} / \mathrm{m}-\mathrm{s}$.

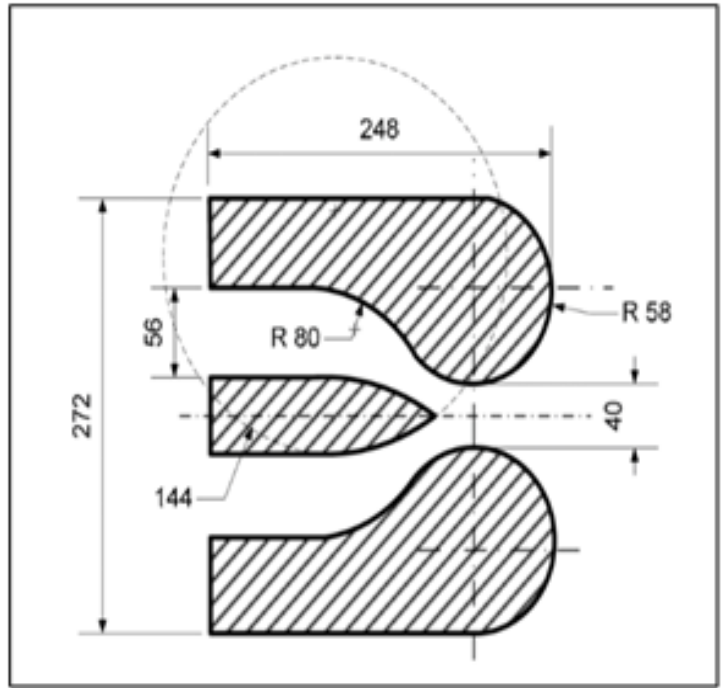

Figura 1(a). Geometria utilizada no problema (Adaptado de Trancossi e Dumas, 2011)

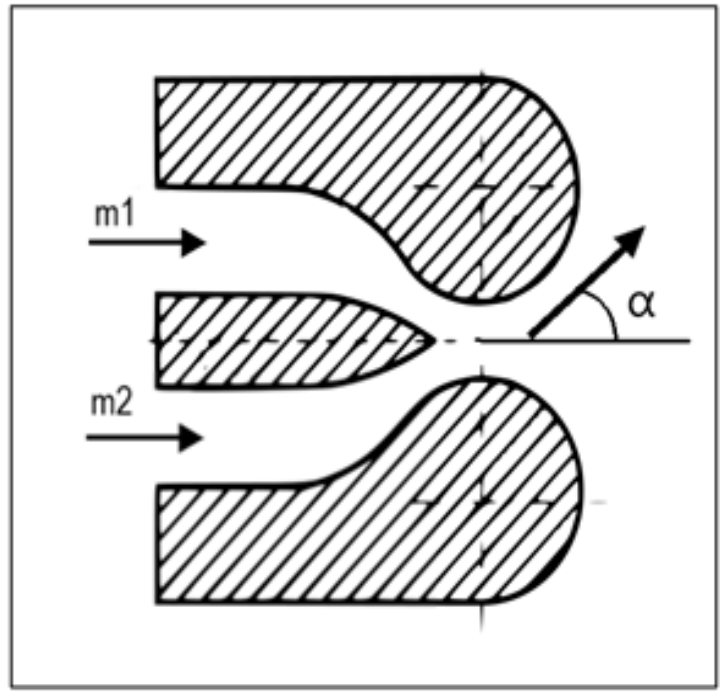

Figura 1(b). Entrada das vazões mássicas e representação do ângulo de deflexão $(\alpha)$

De acordo com Trancossi e Dumas (2011), podemos arranjar as vazões mássicas em uma variável adimensional $m^{*}$, demonstrada por:

$$
m^{*}=\frac{m_{1}-m_{2}}{m_{1}+m_{2}}
$$

Tabela 1. Vazões mássicas utilizadas

\begin{tabular}{lll}
\hline $\boldsymbol{m}^{*}$ & $\dot{\boldsymbol{m}}_{\mathbf{1}}$ & $\dot{\boldsymbol{m}}_{\mathbf{2}}$ \\
\hline 0,00 & 4,0 & 4,0 \\
0,10 & 4,4 & 3,6 \\
0,20 & 4,8 & 3,2 \\
0,25 & 5,0 & 3.0 \\
0,50 & 6,0 & 2,0 \\
0,75 & 7,0 & 1,0 \\
\hline
\end{tabular}


Para a abordagem dos escoamentos turbulentos, incompressíveis, bidimensionais e no regime permanente são utilizadas as equações de conservação de massa e quantidade de movimento médias no tempo (Wilcox, 2006), dadas por:

$$
\begin{array}{r}
\frac{\partial \bar{u}_{i}}{\partial x_{i}}=0 \\
\frac{\partial}{\partial x_{i}}\left(\rho \bar{u}_{i} \bar{u}_{j}\right)=-\frac{\partial \bar{p}}{\partial x_{j}}+\frac{\partial}{\partial x_{j}}\left[\mu\left(\frac{\partial u_{j}}{\partial x_{i}}+\frac{\partial u_{j}}{\partial x_{i}}\right)\right]+\frac{\partial}{\partial x_{j}}\left(-\rho \overline{u^{\prime}{ }_{\imath} u_{j}^{\prime}}\right)
\end{array}
$$

onde $u_{\mathrm{i}}$ representa a velocidade na direção $i$ ( $i=1$ ou 2 , representando a direção $x$ e $y$ do escoamento), $p$ representa a pressão e a barra representa o operador de média temporal. As tensões de Reynolds, oriundas do problema de fechamento, podem ser relacionadas à taxa de deformação por:

$$
\overline{u^{\prime}{ }_{l} u^{\prime}}=\frac{\mu_{T}}{\rho}\left(\frac{\partial \bar{v}_{l}}{\partial x_{j}}+\frac{\partial \bar{v}_{J}}{\partial x_{i}}\right)-\frac{2}{3} k \delta_{i j}
$$

Logo, a viscosidade, representada por $\mu_{\mathrm{T}}$, pode ser descrita para o modelo $k-\varepsilon$ (utilizado no presente trabalho) através da seguinte expressão (Launder e Spalding, 1972):

$$
\mu_{T}=\rho C_{\mu} \frac{k^{2}}{\varepsilon}
$$

Para obtenção da energia cinética da turbulência $(k)$ e da dissipação da mesma $(\varepsilon)$ é necessária a solução de duas equações de transporte adicionais (modelo a duas equações) dadas por (Versteeg e Malalasekera, 2007):

$$
\begin{gathered}
\frac{\partial}{\partial x_{i}}\left(\rho u_{i} k\right)=\frac{\partial}{\partial x_{i}}\left[\frac{\partial k}{\partial x_{i}}\left(\mu+\frac{\mu_{t}}{\sigma_{k}}\right)\right]+G_{k}-\rho \varepsilon-Y_{M}+\sigma_{K} \\
\frac{\partial}{\partial x_{i}}\left(\rho u_{i} \varepsilon\right)=\frac{\partial}{\partial x_{i}}\left[\frac{\partial \varepsilon}{\partial x_{i}}\left(\mu+\frac{\mu_{t}}{\sigma_{\varepsilon}}\right)\right]+C_{1 \varepsilon} \frac{\varepsilon}{k} G_{k}-C_{2 \varepsilon} \rho \frac{\varepsilon^{2}}{k}+\sigma_{\varepsilon}
\end{gathered}
$$

Nessas equações, $G_{k}$ é a taxa de energia cinética turbulenta, $Y_{M}$ é a contribuição das flutuações da turbulência para a taxa de dissipação total. As constantes a serem definidas são $\sigma_{k}$ e $\sigma_{\varepsilon}$ sendo, nesse caso, utilizados os valores padrão. A Tabela 2 mostra o valor das constantes a serem utilizadas para resolução das equações.

Tabela 1. Constantes utilizadas na resolução das equações (Versteeg e Malalasekera, 2007)

\begin{tabular}{ccccc}
\hline$C_{\mu}$ & $C_{1 \varepsilon}$ & $C_{2 \varepsilon}$ & $\sigma_{\varepsilon}$ & $\sigma_{K}$ \\
\hline 0,09 & 1,44 & 1,92 & 1,3 & 1,0 \\
\hline
\end{tabular}

A malha utilizada no problema é feita de aproximadamente 40.000 volumes triangulares. Para captar mais adequadamente os intensos gradientes de velocidades nas regiões de parede, foi realizado um refinamento próximo as mesmas e empregada uma malha com volumes retangulares. As Figs. $2 \mathrm{a}$ e $2 \mathrm{~b}$ mostram a malha utilizada no domínio e um detalhe perto da região de parede, onde se esperam os maiores gradientes de velocidade e pressão. Além disso, foi empregada uma função de parede do tipo Enhanced Wall Treatment devido a maior dificuldade do modelo $\mathrm{k}-\varepsilon$ em predizer os campos em regiões anisotrópicas. 


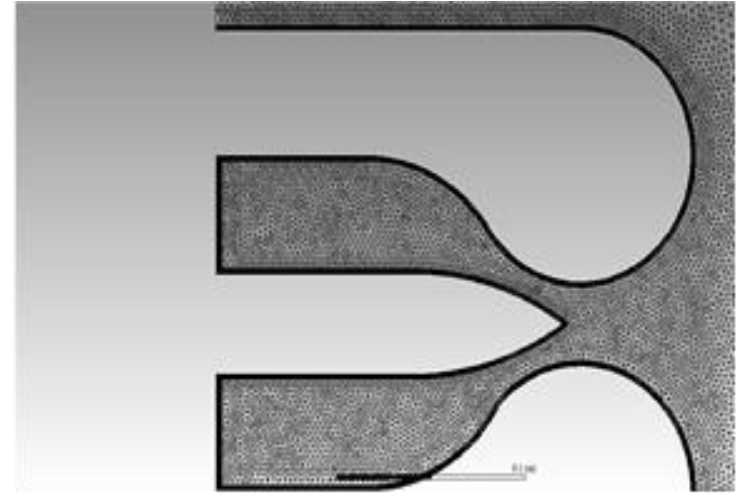

Figura 2(a). Malha utilizada no problema

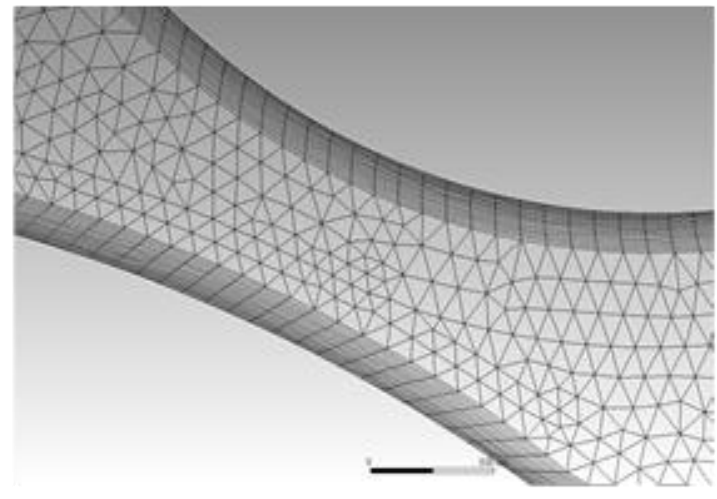

Figura 2(b). Detalhe da região da parede

Para a solução numérica das equações de conservação de massa, quantidade de movimento médias no tempo e as equações de transportes adicionais foi empregado o método dos volumes finitos (MVF), mais precisamente usando o código do software FLUENT (FLUENT, 2011). Para tratamento dos termos advectivos foi empregado o esquema de advecção Upwind e foi utilizado para acoplamento pressão-velocidade o método SIMPLEC. Com relação aos resíduos, foram consideradas convergidas as simulações quando os resíduos de massa, velocidade em $x$ e $y$ e equações de transporte foram inferiores a $10^{-6}$.

Como parte dos resultados do presente trabalho, em um primeiro momento será feito um estudo de independência de malha, para determinar o refinamento adequado da malha para as simulações, visando diminuir o esforço computacional sem ter perda na precisão dos resultados. Posteriormente, será avaliado se para os casos estudados a presente metodologia conduz a resultados que atendam o princípio da conservação de massa no dispositivo. Em seguida, é realizada uma comparação do ângulo de deflexão do escoamento no bocal de saída em função da diferença de vazão mássica entre as duas entradas do dispositivo. Finalmente, compara-se os resultados obtidos com os previamente preditos na literatura por Trancossi e Dumas (2011).

\section{Resultados e discussões}

Primeiramente, um teste de independência de malha foi realizado. Para tal, foram utilizadas malhas com aproximadamente $10.000,20.000,40.000$ e 80.000 volumes finitos. A variável analisada nas simulações foi o ângulo de deflexão causado pela diferença das vazões mássicas nos canais superior e inferior para o caso com $m^{*}=0,1$. A Figura 3 mostra o ângulo de deflexão em função de $m^{*}$, onde é possível observar um comportamento assintótico se formando a partir de 40.000 volumes, com uma variação de apenas 3,33\% no resultado, quando comparado ao próximo refinamento de malha, de 80.000 volumes. Dessa maneira, optou-se por utilizar a malha de 40.000 volumes, já que seria possível obter resultados com erros relativamente insignificantes dispendendo de um menor esforço computacional.

Ainda, no que diz respeito ao ângulo de deflexão, foi criada uma superfície de controle na saída do jato e, dessa maneira, monitorada as variações do ângulo da velocidade e magnitude da velocidade ao longo das iterações, garantindo, assim, que o problema havia convergido. É interessante ressaltar que a convergência das variáveis analisadas ocorreu antes dos valores dos resíduos atingirem o valor especificado, ou seja, não houve variação do ângulo de deflexão e da velocidade após certo número de iterações.

No que diz respeito a consistência referente a conservação de massa do problema, foi comparada a vazão mássica de entrada com a da saída no bocal para identificar se o presente modelo está sendo conservativo. A Tab. 3 mostra as vazões mássicas obtidas na entrada e saída do dispositivo.

A pequena diferença encontrada nas vazões mássicas se deve ao fato de a superfície de controle na saída estar levemente deslocada em relação a saída real do dispositivo. Apesar disso, desvios máximos de aproximadamente 0.2 $\%$ foram encontrados, o que de forma geral indica que o método está sendo conservativo. 


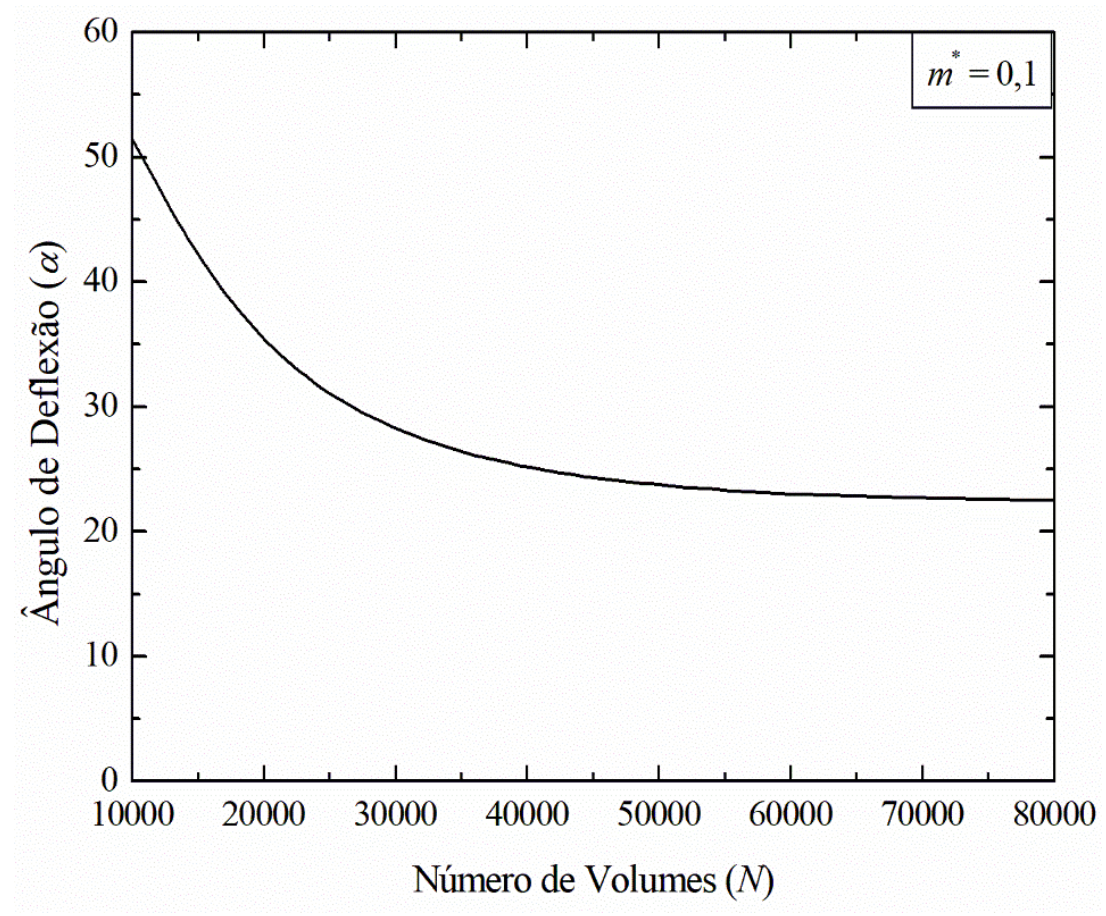

Figura 3. Estudo de independência de malha aplicado ao ângulo de deflexão do escoamento para o caso com $m^{*}=0.1$.

Tabela 3. Vazões mássicas na entrada $\left(m_{\mathrm{e}}\right)$, saída $\left(m_{\mathrm{s}}\right)$ e soma das vazões.

\begin{tabular}{cccc}
\hline $\boldsymbol{m}^{*}$ & $\boldsymbol{m}_{\mathrm{e}}(\mathbf{k g} / \mathbf{s})$ & $\boldsymbol{m}_{\mathbf{s}}(\mathbf{k g} / \mathbf{s})$ & $\boldsymbol{m}_{\mathrm{e}}-\boldsymbol{m}_{\mathbf{s}}(\mathbf{k g} / \mathbf{s})$ \\
\hline 0 & 8 & 8,017 & $-0,017$ \\
0,1 & 8 & 8,017 & $-0,017$ \\
0,2 & 8 & 8,012 & $-0,012$ \\
0,25 & 8 & 8,011 & $-0,011$ \\
0,5 & 8 & 8,004 & $-0,004$ \\
0,75 & 8 & 8,002 & $-0,002$ \\
\hline
\end{tabular}

A Figura 4 mostra os campos de velocidades obtidos nas simulações, onde é possível observar que, com o aumento da diferença entre as vazões mássicas nos canais, o ângulo de deflexão do jato na saída do bocal aumenta. Esse efeito é esperado, já que, aumentando a vazão mássica sem alterar a área da superfície pela qual essa vazão atravessa, aumenta-se a velocidade. De acordo com o Efeito Coanda, o fluído com maior velocidade tende a arrastar o fluido com menor velocidade, o que pode ser claramente observado nas imagens.

Comparando os resultados obtidos com os resultados publicados por Trancossi e Dumas (2011), é possível verificar que os resultados são bem aproximados e seguem um mesmo padrão de crescimento, conforme pode ser observado na Fig. 5. 

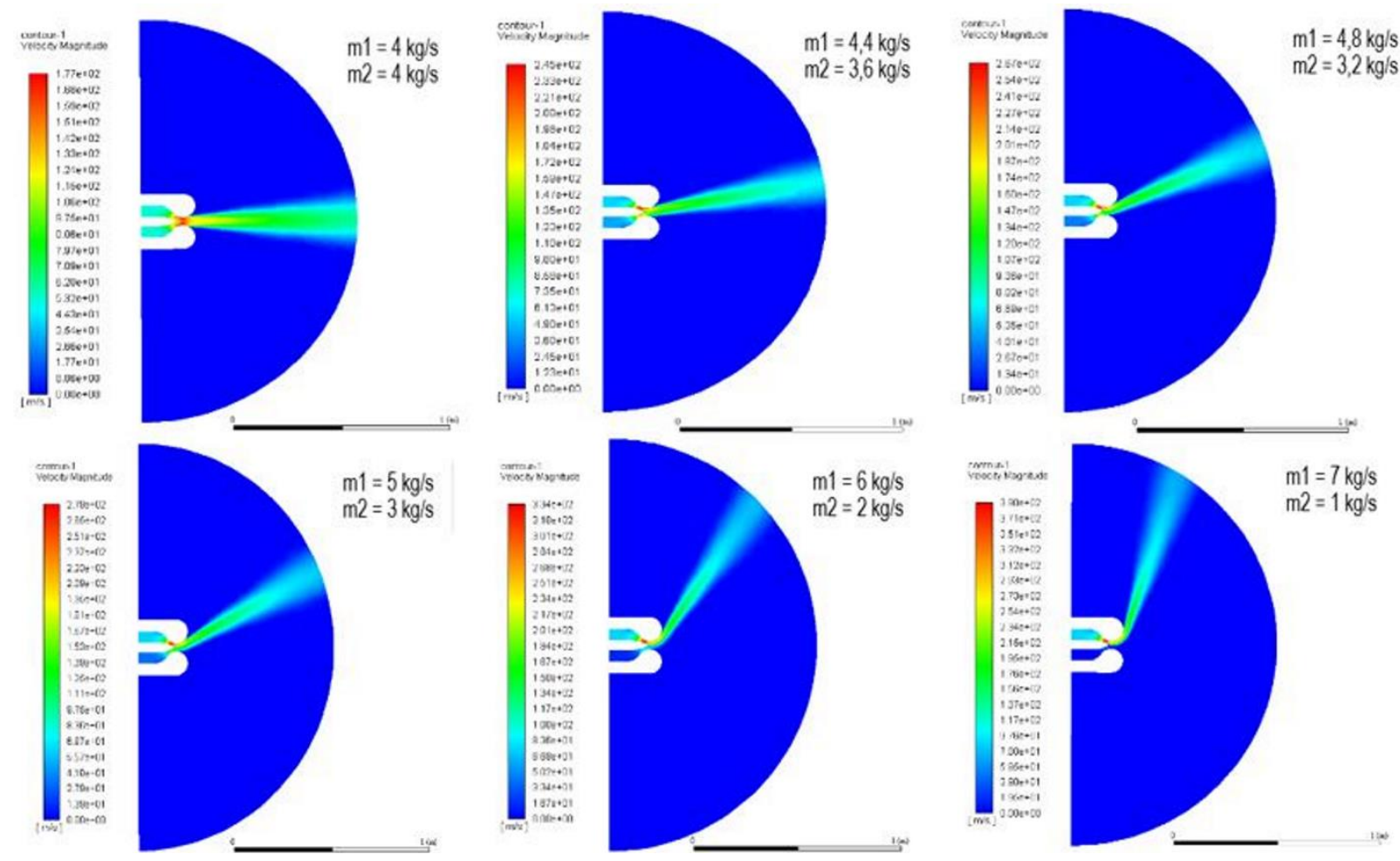

Figura 4. Gradientes de velocidade para todos $\mathrm{m}^{*}$ simulados.

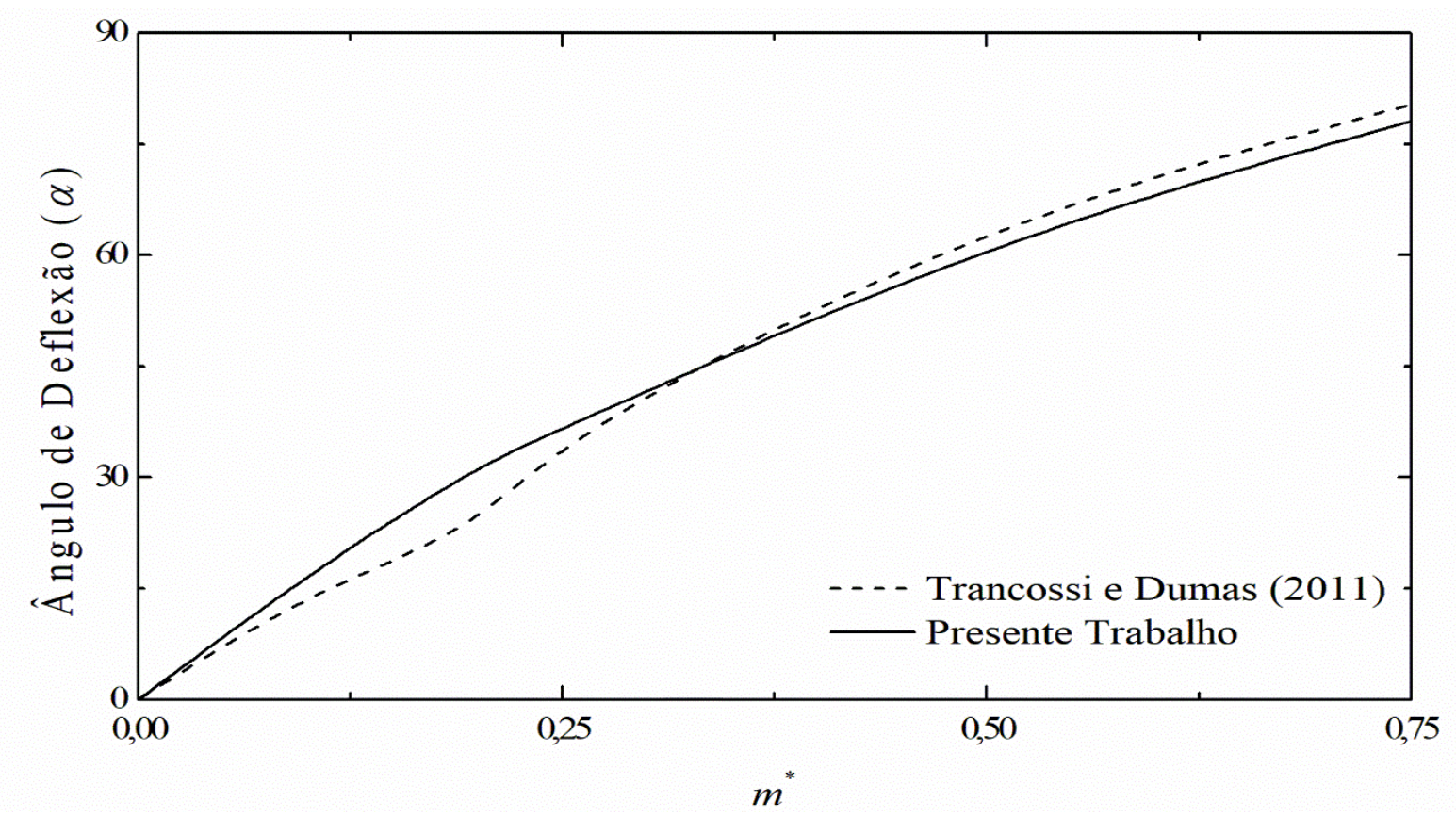

Figura 5. Comparação dos resultados obtidos nas simulações com os resultados obtidos por Trancossi e Dumas (2011).

De uma forma geral, a diferença nos resultados é pequena, com exceção do caso $m^{*}=0.2$ onde uma diferença de aproximadamente $30 \%$ foi observada. Apesar desse ponto com desvio, a tendência da curva é muito similar e os demais pontos apresentam desvios inferiores a $4.0 \%$, o que verifica o presente modelo numérico. 0 desvio encontrado também pode estar associado a apresentação dos resultados no trabalho de Trancossi e Dumas (2011) ter sido realizada na forma de ajuste de uma curva senoidal com ângulo de deflexão em função de $m^{*}$, similarmente a: 


$$
\theta(m *)=a+b \sin \left(c m^{*}+d\right)
$$

Um ajuste similar foi realizado no presente trabalho a fim de comparar com a curva ajustada apresentada na literatura. A Fig. 6 ilustra a comparação entre as curvas obtidas no presente trabalho com o apresentado em Trancossi e Dumas (2011). Acredita-se que o desvio encontrado na Fig. 7 se deve a geometria utilizada no presente trabalho. A inserção do septo na zona entre as superfícies curvas do bocal utilizadas por Trancossi e Dumas não foi informada na literatura, ocasionando uma pequena divergência entre os modelos testados e, consequentemente, o desvio nos resultados obtidos. Além disso, Trancossi e Dumas não apresentaram o teste de independência de malha, o que pode acarretar em resultados errôneos por conta de uma malha não suficientemente refinada.

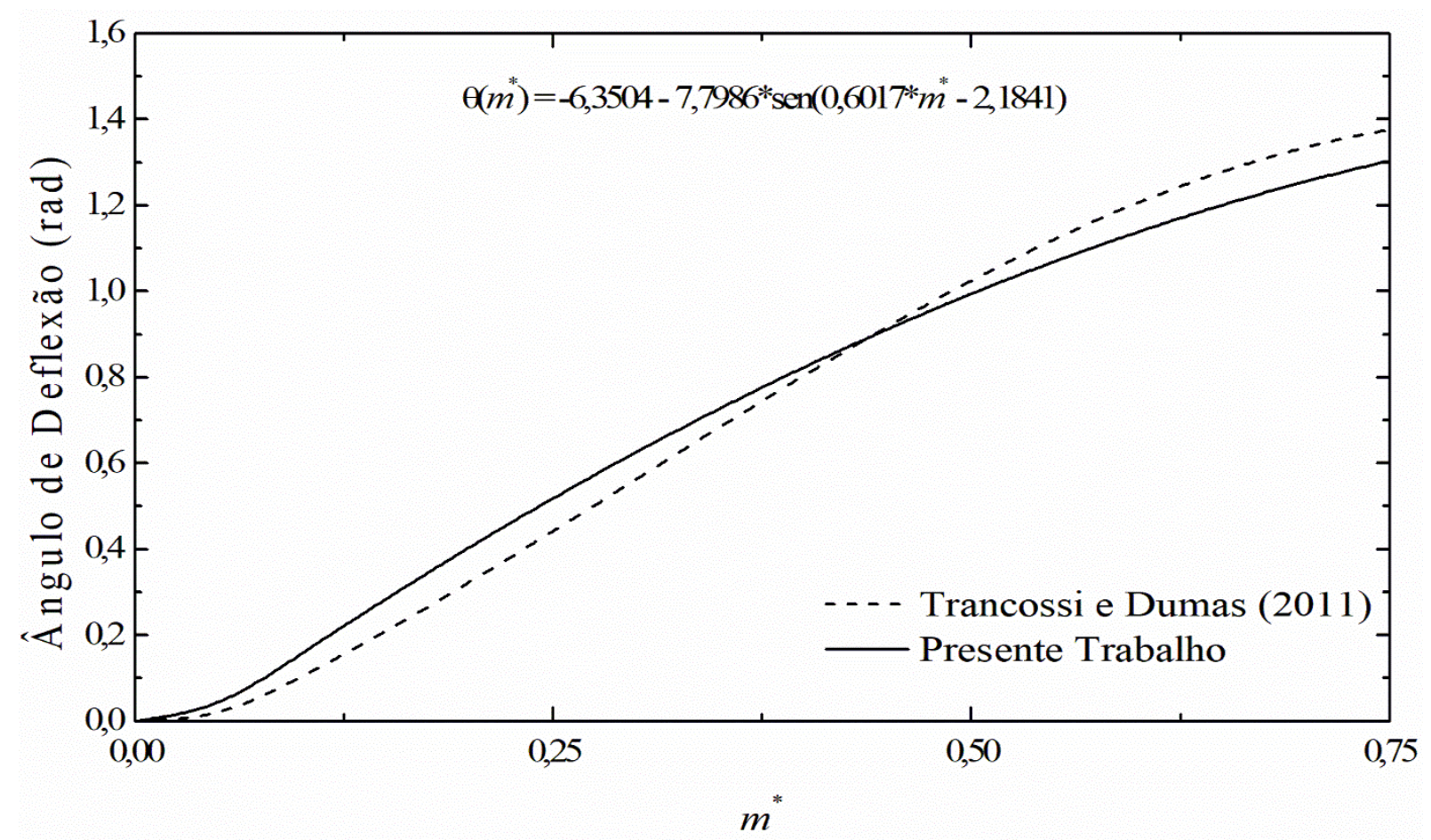

Figura 6. Comparação do ajuste de curva na forma senoidal, em função de $\mathrm{m}^{*}$, utilizando uma sobrepressão de 4000Pa na parede.

\section{Conclusão}

No presente trabalho foi desenvolvido um modelo numérico para a simulação de escoamentos turbulentos, incompressíveis e permanentes em uma geometria que simula um bocal do tipo H.O.M.E.R. que pode ser empregado como propulsor e/ou dispositivo de manobra em aplicações aeronáuticas e navais. O modelo numérico desenvolvido resolve as equações de conservação de massa e quantidade de movimento médias no tempo (RANS) através do método dos volumes finitos. Para o fechamento da turbulência é empregado o modelo $k-\varepsilon$.

Em um primeiro teste foi identificado que o modelo desenvolvido atende ao princípio da conservação de massa. Posteriormente, foi verificada a influência da diferença de magnitudes nas vazões mássicas nas duas entradas do dispositivo sobre o ângulo de deflexão do escoamento na saída do bocal. Foi observado que o aumento da razão entre as vazões mássicas da entrada $1\left(m_{1}\right)$ e entrada $2\left(m_{2}\right)$ conduziram a um aumento no ângulo de deflexão do jato na saída do bocal. Os resultados foram comparados com os apresentados em Trancossi e Dumas (2011). Apesar de ocorrer um desvio nas magnitudes obtidas para $m^{*}=0.2$, os resultados apresentaram uma tendência bastante similar e as magnitudes dos ângulos de deflexão foram concordantes (erro máximo de $4 \%$ ) nos demais pontos analisados. Uma causa provável para o desvio é o emprego de uma geometria levemente diferente no modelo testado por falta de informações exatas. Além disso, a ausência de um teste de independência de malha pode ter ocasionado resultados não tão precisos na solução do modelo apresentado por Trancossi e Dumas. 
Para trabalhos futuros, sugere-se um estudo mais aprofundado da influência da posição do septo no ângulo de deflexão gerado. Além disso, o desenvolvimento de um estudo experimental que permita também uma validação do presente modelo numérico.

Agradecimentos: $O$ autor Y. B. El Halal agradece a FAPERGS pela bolsa de iniciação científica. O autor R. de L. Lemos agradece ao CNPq pela bolsa de mestrado. Os autores E. D. dos Santos, L. A. Isoldi e L. A. O. Rocha agradecem ao CNPq pelas bolsas de produtividade em pesquisa (Processos: 306024/2017-9, 306012/2017-0, 307847/2015-2).

\section{Referências}

1. Andersson, B., Andersson, R., Håkansson, L., Mortensen, M., Sudiyo, R., \& Van Wachem, B. (2011). Computational fluid dynamics for engineers. Cambridge University Press.

2. Ansys Fluent (2011). Release 14.0. [Computer software].

3. Chapman, L. (2007). Transport and climate change: a review. Journal of Transport Geography, 15(5), 354-367. https://doi.org/10.1016/j.jtrangeo.2006.11.008

4. Coanda, H. (1936). U.S. Patent No. 2,052,869. Washington, DC: U.S. Patent and Trademark Office.

5. Dumas, A., Pascoa, J., Trancossi, M., Tacchini, A., Ilieva, G., \& Madonia, M. (2012). Acheon Project: A Novel Vectoring Jet Concept (p. 499). ASME. https://doi.org/10.1115/IMECE2012-87638

6. Johnson, T. V. (2009). Diesel emission control in review. SAE international journal of fuels and lubricants, 1(1), 68-81.

7. Launder, B. E., \& Spalding, D. B. (1972). Mathematical models of turbulence (No. BOOK). Academic press.

8. Panton, R. L. (2013). Incompressible Flow (4 $4^{\circ}$ ed). Wiley.

9. Páscoa, J., Dumas, A., Trancossi, M., Stewart, P., \& Vucinic, D. (2013). A review of thrust-vectoring in support of a $\mathrm{V} / \mathrm{STOL}$ non-moving mechanical propulsion system. Open Engineering, 3(3). https://doi.org/10.2478/s13531-013-0114-9

10. Rodrigues, F., Páscoa, J. C., Dumas, A., \& Trancossi, M. (2013). Preliminary design, set-up and testing of a plasma DBD actuator for boundary layer control. International Conference on Engineering UBI.

11. Rose, M. G., Harvey, N. W., Seaman, P., Newman, D. A., \& McManus, D. (2001, June). Improving the Efficiency of the Trent 500 HP Turbine Using Non-Axisymmetric End Walls: Part II-Experimental Validation. In ASME Turbo Expo 2001: Power for Land, Sea, and Air (pp. V001T03A081-V001T03A081). American Society of Mechanical Engineers.Launder, B. E., \& Spalding, D. B. (1972). Mathematical models of turbulence (No. BOOK). Academic press.

12. Trancossi, M., \& Dumas, A. (2011). Coanda synthetic jet deflection apparatus and control (No. 2011-01-2590). SAE Technical Paper.

13. Wilcox, D.C. Turbulence Modeling for CFD, third ed., DCW Industries, 2006.

14. Versteeg, H. K., \& Malalasekera, W. (2007). An Introduction to Computational Fluid Dynamics - The Finite Volume Method ( $2^{\circ}$ ed). England: Pearson Education. 